\title{
The Periodic Table of Elementary Particles for Baryonic Matter and Dark Matter: Upward-Going ANITA Events
}

\author{
Ding-Yu Chung \\ Utica, Michigan, USA \\ Email: dy_chung@yahoo.com
}

How to cite this paper: Chung, D.-Y. (2018) The Periodic Table of Elementary Particles for Baryonic Matter and Dark Matter: Upward-Going ANITA Events. Journal of Modern Physics, 9, 2308-2319. https://doi.org/10.4236/jmp.2018.913146

Received: October 10, 2018

Accepted: November 4, 2018

Published: November 7, 2018

Copyright $\odot 2018$ by author and Scientific Research Publishing Inc. This work is licensed under the Creative Commons Attribution International License (CC BY 4.0).

http://creativecommons.org/licenses/by/4.0/

\section{(c) (i) Open Access}

\begin{abstract}
This paper posits that the upward-going ANITA events are derived from the cosmic ray of the baryonic-dark matter (BDM) Higgs boson. In the extended standard model (ESM) for baryonic matter and dark matter, the spontaneous symmetry breaking through the Higgs mechanism for the symmetrical massless baryonic matter left-handed neutrinos and massless dark matter right-handed neutrinos produced massless baryonic matter left-handed neutrinos, sterile massive dark matter neutrinos, and the BDM Higgs boson. The BDM Higgs boson is the composite of the high-mass tau neutrino and the high-mass dark matter neutrino. During the passage through the high-density part of the Earth, the BDM Higgs boson is transformed into the oscillating BDM Higgs boson between the composite of the high-mass tau neutrino and the high-mass dark matter neutrino and the composite of the high-mass tau neutrino and the low-mass dark matter neutrino. The oscillating BDM Higgs boson decays into the high-mass tau neutrino with the extra energy and the low-mass dark matter neutrino $(27 \mathrm{eV})$ in the low-density water-ice layer of the Earth. The high-mass tau neutrino is converted into ultra-high-energy tau neutrino which decays into tau lepton through the charged-current interactions, and tau lepton emerges from the surface of ice. Based on the periodic table of elementary particles, the calculated value for the high-mass tau neutrino with the extra energy is $0.47 \mathrm{EeV}$ in good agreement with the observed 0.56 and $0.6 \mathrm{EeV}$. The periodic table of elementary particles for baryonic matter, dark matter, and gravity is based on the seven principal mass dimensional orbitals for stable baryonic matter leptons (electron and left-handed neutrinos), gauge bosons, gravity, and dark matter and the seven auxiliary mass dimensional orbitals for unstable leptons (muon and tau) and quarks, and calculates accurately the masses of all elementary particles and the cosmic rays by using only five known constants.
\end{abstract}




\section{Keywords}

ANITA, Periodic Table of Elementary Particles, Higgs Boson, Baryonic-Dark Matter Higgs Boson, Cosmic Rays, Upward-Going, Dark Matter, Baryonic Matter, Extended Standard Model

\section{Introduction}

The Antarctic Impulsive Transient Antenna (ANITA) experiment [1] is established to the detection of the cosmogenic ultra-high-energy (UHE) neutrinos at the scale of EeV. The three balloon flights of the ANITA experiment have produced two unusual steeply upward-going cosmic ray events with energies of $\approx 0.6$ $\mathrm{EeV}$ [2] and $\approx 0.56 \mathrm{EeV}$ [3]. These shower events have the characteristics of the decay of a tau lepton, which emerges from the surface of the ice, and the tau lepton is explained as the product of a UHE parent tau neutrino by the charged-current interactions with the Earth matter. However, such UHE tau neutrino cannot survive the passage through the Earth. The explanations for such anomalous events include the sterile neutrino origin [4] [5], the decay of the quasi-stable dark matter in the Earth's core [6] [7], and supersymmetric stau slepton [8].

This paper posits that the upward-going ANITA events are derived from the cosmic ray of the baryonic-dark matter (BDM) Higgs boson that travels through the Earth. In the standard model (SM) for baryonic matter, the SM spontaneous symmetry breaking through the Higgs mechanism for the symmetrical massless electromagnetism and massless weak interaction produced massless photon, massive weak bosons, and the standard model Higgs boson. (The standard model classifies all known elementary particles for baryonic matter, describes the electromagnetic, weak, and strong interactions, and does not include dark matter and the gravitational force.) Equally, in the extended standard model (ESM) for baryonic matter, dark matter, and gravity, the BDM spontaneous symmetry breaking through the Higgs mechanism for the symmetrical massless baryonic matter left-handed neutrinos and massless dark matter right-handed neutrinos produced massless baryonic matter left-handed neutrinos, sterile massive dark matter neutrinos, and the BDM Higgs boson. Dark matter particles are the sterile massive neutrinos. This paper proposes the BDM Higgs boson is the composite of the high-mass tau neutrino and the high-mass dark matter neutrino. Other than gravity, sterile dark matter does not undergo any interaction with baryonic matter. Meanwhile, dark matter is incompatible to dense baryonic matter [9], because the incompatibility explains the failure to detect dark matter by the contact (interaction) between dark matter and baryonic matter on the Earth. The BDM Higgs boson is both baryonic matter and dark matter. The inactive dark matter in the BDM Higgs boson allows the BDM Higgs boson to be stable (inactive) in space, air, water, and ice. However, during the passage through the high-density part of the Earth, because of the inactivity and the incompati- 
bility between dark matter and dense baryonic matter, the BDM Higgs boson is transformed into the oscillating BDM Higgs boson between the composite of the high-mass tau neutrino and the high-mass dark matter neutrino and the composite of the high-mass tau neutrino and the low-mass dark matter neutrino to maintain stability (inactivity) with the maximum dark matter (the high-mass dark energy neutrino) and to minimize incompatibility with the minimum dark matter (the low-mass dark matter neutrino). Near the end of the passage through the Earth, the low-density water-ice layer of the Earth allows the dark matter in the oscillating BDM Higgs boson to escape, so the oscillating BDM Higgs boson decays into the high-mass tau neutrino with the extra energy and the incompatible low-mass dark matter neutrino $(27 \mathrm{eV})$ which is easier to escape than the high-mass dark matter neutrino. The high-mass tau neutrino is converted into ultra-high-energy tau neutrino which decays into tau lepton through the charged-current interactions, and tau lepton emerges from the surface of ice. As shown later in the paper, based on the periodic table of elementary particles [10] [11], the calculated mass for the high-mass tau neutrino with the extra energy is $0.47 \mathrm{EeV}$ in the good agreement with the observed 0.6 and 0.56 $\mathrm{EeV}[2]$ [3].

In the ESM to include baryonic matter, dark matter, and gravity, the seven extra spacetime dimensions in the 11 spacetime dimensional membrane in $\mathrm{M}$-theory are in the form of the seven mass dimensional orbitals for all internal symmetries of elementary particles [10] [11]. The two sets of the seven mass dimensional orbitals are the seven principal mass dimensional orbitals for stable baryonic leptons (electron and left-handed neutrinos), gauge bosons, gravity, and dark matter and the seven auxiliary mass dimensional orbitals for unstable leptons (muon and tau) and quarks. These two seven mass dimensional orbitals generate the periodic table of elementary particles and the ESM for baryonic matter, dark matter, and gravity. The periodic table of elementary particles calculates accurately the masses of all elementary particles and the cosmic rays by using only five known constants [12] [13].

The formation of the periodic table of elementary particles involves the three steps. The first step is the BDM spontaneous symmetry breaking through the Higgs mechanism for the symmetrical five massless baryonic matter left-handed neutrinos and five massless dark matter right-handed neutrinos on the principal mass dimensional orbitals to produce massless baryonic matter left-handed neutrinos, sterile massive dark matter neutrinos, and the BDM Higgs boson. The second step is the addition of electromagnetism as $\mathrm{U}(1)_{\mathrm{EM}}$ and the standard model spontaneous symmetry breaking $\left(\mathrm{SU}(2)_{\mathrm{L}} \times \mathrm{U}(1)_{\mathrm{Y}} \rightarrow \mathrm{U}(1)_{\mathrm{EM}}\right)$ through the Higgs mechanism to produce massless neutrinos-massive weak bosons, massive electron-massless photon, and the standard model Higgs boson. The third step is the addition of the seven auxiliary mass dimensional orbitals for unstable leptons (muon and tau) and quarks to form the periodic table of elementary particles. Section 2 describes the BDM spontaneous symmetry breaking for baryo- 
nic matter neutrinos and dark matter neutrinos. Section 3 describes the addition of $U(1)$, the standard model spontaneous symmetry breaking, and the upward-going ANITA events. Section 4 describes the period table of elementary particles for baryonic matter and dark matter and the cosmic rays.

\section{The BDM Spontaneous Symmetry Breaking for Symmetrical Baryonic Matter and Dark Matter}

The first step in the formation of the periodic stable of elementary particles is the BDM spontaneous symmetry breaking for baryonic matter and dark matter. As described in the previous paper [14], there are one type of baryonic matter and five types of dark matter, resulting in the mass ratio of dark matter to baryonic matter as 5 to 1 in the good agreement with the observation [15]. Initially, the symmetry $\mathrm{U}(1)_{\mathrm{L}} \times \mathrm{U}(1)_{\mathrm{R}}$ between baryonic matter and dark matter in each mass dimensional orbital provided the symmetrical distribution between baryonic matter as the five massless left-handed neutrinos $\left(v_{\mathrm{L} 5}, v_{\mathrm{L} 6}, v_{\mathrm{L} 7}, v_{\mathrm{L} 8}\right.$, and $\left.v_{\mathrm{L} 9}\right)$ and dark matter as the five massless right-handed neutrinos $\left(v_{\mathrm{R} 5}, v_{\mathrm{R} 6}, v_{\mathrm{R} 7}, v_{\mathrm{R} 8}\right.$, and $\left.v_{\mathrm{R} 9}\right)$ on the principal mass dimensional orbitals from $d$ (mass dimensional orbital number) $=5$ to $d=9$ as in Figure 1 .

Each mass dimensional orbital had $\mathrm{U}(1)_{\mathrm{L}} \times \mathrm{U}(1)_{\mathrm{R}}$ symmetry. The spontaneous symmetry breaking through the Higgs mechanism for the symmetrical massless baryonic matter left-handed neutrinos and massless dark matter right-handed neutrinos produced four massless baryonic matter left-handed neutrinos $\left(v_{\mathrm{L} 5}\right.$, $v_{\mathrm{L} 6}, v_{\mathrm{L} 7}$, and $\left.v_{\mathrm{L} 8}\right)$ one massive baryonic matter left-handed neutrino $\left(v_{\mathrm{L} 9}\right)$, five sterile massive dark matter neutrinos ( $v_{\mathrm{DM} 5}, v_{\mathrm{DM} 6}, v_{\mathrm{DM} 7}, v_{\mathrm{DM} 8}$, and $\left.v_{\mathrm{DM} 9}\right)$, and the BDM Higgs boson. In each mass dimensional orbital, the spontaneous symmetry breaking involved $\mathrm{U}(1)_{\mathrm{L}} \times \mathrm{U}(1)_{\mathrm{R}} \rightarrow \mathrm{U}(1)_{\mathrm{L}}$. The exception is $v_{\mathrm{L} 9}$ which was massive because $v_{\mathrm{L} 9}$ was a part of the massive BDM Higgs boson.

The seven mass dimensional orbitals are arranged as $\mathrm{F}_{5} \mathrm{~B}_{5} \mathrm{~F}_{6} \mathrm{~B}_{6} \mathrm{~F}_{7} \mathrm{~B}_{7} \mathrm{~F}_{8} \mathrm{~B}_{8} \mathrm{~F}_{9}$ $B_{9} F_{10} B_{10} F_{11} B_{11}$, where $F_{d}$ and $B_{d}$ are mass dimensional fermion and mass dimensional boson, respectively. As described in the previous papers [10] [11], the masses of massive dark matter neutrinos are related to each other with three simple formulae as the follows.

$$
M_{\mathrm{d}, B}=M_{\mathrm{d}, F} / \alpha_{\mathrm{d}}
$$

Massless baryonic matter left-handed neutrinos

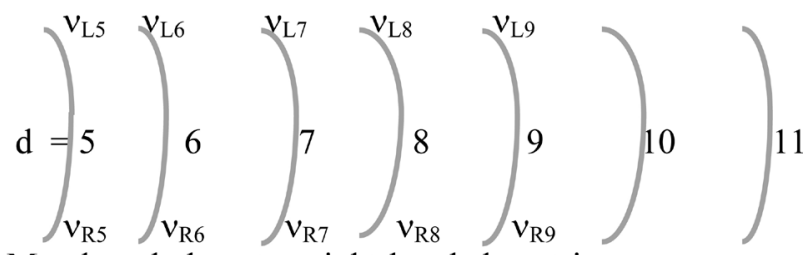

Massless dark matter right-handed neutrinos

Figure 1. Baryonic matter neutrinos and dark matter neutrinos on the principal mass dimensional orbitals where $d=$ principal mass dimensional orbital numbers from $d=5$ to $\mathrm{d}=11$. 


$$
\begin{aligned}
& M_{\mathrm{d}+1, \mathrm{~F}}=M_{\mathrm{d}, B} / \alpha_{\mathrm{d}+1} \\
& M_{\mathrm{d}+1, \mathrm{~B}}=M_{\mathrm{d}, B} / \alpha_{\mathrm{d}+1}^{2},
\end{aligned}
$$

where $\mathrm{d}$ is the mass dimensional orbital number, $\mathrm{F}$ is fermion, and $\mathrm{B}$ is boson. Each dimension has its own $\alpha_{\mathrm{d}}$, and all $\alpha_{d}$ 's except $\alpha_{7}\left(\alpha_{w}\right)$ of the seventh dimension (weak interaction) are equal to $\alpha$, the fine structure constant of electromagnetism. The given observed masses are the mass of electron for $\mathrm{F}_{6}$ and the mass of $\mathrm{Z}$ boson for $\mathrm{B}_{7}$. From Equations (1) and (3), $\alpha_{\mathrm{w}}=\alpha_{7}=\alpha$ of week interaction = $\left(M_{B 6} M_{B 7}\right)^{1 / 2}=\left(M_{F 6} / \alpha / M_{B 7}\right)^{1 / 2}=\left(M_{e} / \alpha / M_{Z}\right)^{1 / 2}=0.02771$. Therefore, the masses of dark matter neutrinos are as in Table 1.

\section{The Addition of U(1), the Standard Model Spontaneous Symmetry Breaking, and the Upward-Going ANITA Events}

In the second step in the formation of the periodic table of elementary particles, electromagnetism as massless $\mathrm{U}(1)_{\mathrm{EM}}$ was added to $v_{\mathrm{L} 6}$ to become election which formed massless $\mathrm{SU}(2)_{\mathrm{L}} \times \mathrm{U}(1)_{\mathrm{Y}}$ with baryonic matter neutrino, and then the standard model spontaneous symmetry breaking involved $\mathrm{SU}(2)_{\mathrm{L}} \times \mathrm{U}(1)_{\mathrm{Y}}$ $\rightarrow \mathrm{U}(1)_{\mathrm{EM}}$ through the Higgs mechanism to produce massive electron-massless photon, massless neutrino-massive weak bosons, and the standard model Higgs boson as in Figure 2 and Table 2.

The standard model spontaneous symmetry breaking generated the observed stable baryonic leptons (electron and left-handed neutrinos) which follow the standard model (SM) where electron neutrino, muon neutrino, and tau neutrino are massless. The extended standard model (ESM) includes an additional neutrino

Table 1. The masses of initial baryonic matter neutrinos and dark matter neutrinos.

\begin{tabular}{cccccc}
\hline $\mathrm{F}_{\mathrm{d}}$ & stable baryonic matter leptons & mass $(\mathrm{eV})$ & dark matter leptons & mass & $\mathrm{eV}$ (calculated) \\
\hline $\mathrm{F}_{5}$ & $v_{\mathrm{L} 5}$ & massless & $v_{\mathrm{DM} 5}$ & $\mathrm{M}_{\mathrm{e}} \alpha^{2}$ & 27 \\
$\mathrm{~F}_{6}$ & $v_{\mathrm{L} 6}$ & massless & $v_{\mathrm{DM} 6}$ & $\mathrm{M}_{\mathrm{e}}$ & $5.11 \times 10^{5}$ (given) \\
$\mathrm{F}_{7}$ & $v_{\mathrm{L} 7}$ & massless & $v_{\mathrm{DM} 7}$ & $\mathrm{M}_{\mathrm{Z}} \alpha_{\mathrm{w}}$ & $2.53 \times 10^{9}$ \\
$\mathrm{~F}_{8}$ & $v_{\mathrm{L} 8}$ & massless & $v_{\mathrm{DM} 8}$ & $\mathrm{M}_{\mathrm{Z}} / \alpha$ & $1.25 \times 10^{13}$ \\
$\mathrm{~F}_{9}$ & $v_{\mathrm{L} 9}$ & massive & $v_{\mathrm{DM} 9}$ & $\mathrm{M}_{\mathrm{Z}} / \alpha^{3}$ & $2.35 \times 10^{17}$ \\
\hline
\end{tabular}

$\mathrm{F}=$ fermion, $\mathrm{d}=$ mass dimensional orbital number, $\mathrm{M}_{\mathrm{e}}=$ mass of electron, $\mathrm{M}_{\mathrm{Z}}=$ mass of $\mathrm{Z}$ boson, $\alpha=\alpha_{\mathrm{e}}, \alpha_{w}$ $=0.02771$.

Stable baryonic matter leptons

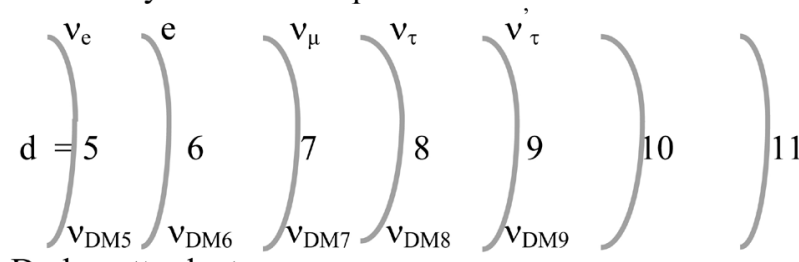

Dark matter leptons

Figure 2. Stable baryonic matter leptons and dark matter leptons in the seven principal mass dimensional orbitals. 
Table 2. The observed masses of stable baryonic leptons and dark matter leptons.

\begin{tabular}{cccccc}
\hline $\mathrm{F}_{\mathrm{d}}$ & $\begin{array}{c}\text { stable baryonic } \\
\text { matter leptons }\end{array}$ & mass $(\mathrm{eV})$ & $\begin{array}{c}\text { dark matter } \\
\text { leptons }\end{array}$ & mass & $\mathrm{eV}$ (calculated) \\
\hline $\mathrm{F}_{5}$ & $v_{\mathrm{e}}$ & massless & $v_{\mathrm{DM} 5}$ & $\mathrm{M}_{\mathrm{e}} \alpha^{2}$ & 27 \\
$\mathrm{~F}_{6}$ & $\mathrm{e}$ & $5.11 \times 10^{5}$ (given) & $v_{\mathrm{DM} 6}$ & $\mathrm{M}_{\mathrm{e}}$ & $5.11 \times 10^{5}$ (given) \\
$\mathrm{F}_{7}$ & $v_{\mu}$ & massless & $v_{\mathrm{DM} 7}$ & $\mathrm{M}_{\mathrm{Z}} \alpha_{\mathrm{w}}$ & $2.53 \times 10^{9}$ \\
$\mathrm{~F}_{8}$ & $v_{\tau}$ & massless & $v_{\mathrm{DM} 8}$ & $\mathrm{M}_{\mathrm{Z}} / \alpha$ & $1.25 \times 10^{13}$ \\
$\mathrm{~F}_{9}$ & $v_{\tau}^{\prime} \quad$ (high-mass $\left.v_{\tau}\right)$ & massive & $v_{\mathrm{DM} 9}$ & $\mathrm{M}_{\mathrm{Z}} / \alpha^{3}$ & $2.35 \times 10^{17}$ \\
& $\begin{array}{c}\text { baryonic-dark } \\
\text { matter Higgs boson }\end{array}$ & massive & $v_{\tau}^{\prime} \overline{\mathrm{DM}}_{\mathrm{DM}}$ & $2 \mathrm{M}_{\mathrm{Z}} / \alpha^{3}$ & $4.7 \times 10^{17}$ \\
\hline
\end{tabular}

$\mathrm{F}=$ dimensional fermion, $\mathrm{d}=$ principal mass dimensional mass orbital number, $\mathrm{M}_{\mathrm{e}}=$ mass of electron, $\mathrm{M}_{\mathrm{Z}}$ $=$ mass of $\mathrm{Z}$ boson, $\alpha=\alpha_{\mathrm{e}}, \alpha_{\mathrm{w}}=\alpha_{7}=\alpha$ of week interaction $=0.02771$.

$v_{\tau}^{\prime}\left(\mathrm{F}_{9}\right)$ as the high-mass tau neutrino for the formation of the BDM Higgs boson which is $v_{\tau}^{\prime} \bar{v}_{\text {DM9 }}$ with the mass of $4.7 \times 10^{17} \mathrm{eV}=0.47 \mathrm{EeV}$ as in Table 2 .

The BDM Higgs boson as the composite of high-mass neutrinos is a decay product of the UHE pion and neutron from the UHE interaction with the cosmic microwave background [16] [17] which is $p+\gamma_{\mathrm{CMB}} \rightarrow p$ (or $\left.n\right)+\mathbf{n} \pi, p+$ $\gamma_{\mathrm{CMB}} \rightarrow \Delta^{+}(1232) \rightarrow p+\pi^{0}$ (or $n+\pi^{+}$) where $\mathbf{n}$ is the total number of the produced $\pi$ 's. Other than gravity, sterile dark matter does not undergo any interaction with baryonic matter. Meanwhile, dark matter is incompatible to dense baryonic matter [9], because the incompatibility explains the failure to detect dark matter by the contact (interaction) between dark matter and baryonic matter on the Earth. As the composite of the high-mass tau neutrino $v_{\tau}^{\prime}$ and the high-mass dark matter neutrino $v_{\mathrm{DM} 9}$, the BDM Higgs boson is both baryonic matter and dark matter. Because of the inactivity (other than gravity) between dark matter and dense baryonic matter, the dark matter in the BDM Higgs boson allows the BDM Higgs boson to be stable and inactive in space, air, water, and ice. However, during the passage through the high-density part of the Earth, because of the the inactivity (other than gravity) and the incompatibility between dark matter and dense baryonic matter, the $\mathrm{BDM}$ boson is transformed into the oscillating BDM Higgs boson between the composite the high-mass tau neutrino and the high-mass dark matter neutrino and the composite the high-mass tau neutrino and the low-mass dark matter neutrino to maintain stability (inactivity) with the maximum dark matter (the high-mass dark matter neutrino) and to minimize incompatibility with the minimum dark matter (the low-mass dark matter neutrino). Near the end of the passage through the Earth, the low-density water-ice layer of the Earth allows incompatible dark matter in the oscillating BDM Higgs boson to escape, so the BDM Higgs boson decays into the high-mass tau neutrino with the extra energy and the incompatible low-mass dark matter neutrino $\bar{v}_{\mathrm{DM} 5}(27 \mathrm{eV})$ which is easier to escape from the oscillating BDM Higgs boson than the high-mass dark matter neutrino $\bar{v}_{\text {DM9 }}$. Free incompatible low-mass dark matter neutrino $\bar{v}_{\mathrm{DM} 5}$ avoids dense baryonic matter, such as the 
detector. The high-mass tau neutrino is converted into ultra-high-energy tau neutrino which decays into tau lepton through the charged-current interactions, and tau lepton emerges from the surface of ice as follows.

$H_{\mathrm{BDM}}=v_{\tau}^{\prime} \bar{\nu}_{D M 9}$ in space, air, water and ice

$$
\begin{aligned}
& \stackrel{\text { transformation in the high-density part of the Earth }}{\longrightarrow} \\
& \text { Oscillating } H_{\mathrm{BDM}} \text { between } v_{\tau}^{\prime} \bar{\nu}_{D M 9} \text { and } v_{\tau}^{\prime} \bar{\nu}_{D M 5} \\
& \stackrel{\text { decay in the water-ice layer }}{\longrightarrow} v_{\tau}^{\prime} \text { with the extra energy }+\bar{v}_{D M 5}(27 \mathrm{eV}) \\
& \stackrel{v_{\tau}^{\prime} \text { with the extra energy } \stackrel{\text { conversion }}{\longrightarrow} \text { ultra-high-energy } v_{\tau}}{\stackrel{\text { charged-current interaction }}{\longrightarrow} \tau,}
\end{aligned}
$$

where $H_{\mathrm{BDM}}$ is the BDM Higgs boson. The decay products of tau lepton were detected by the ANITA. The calculated mass (Table 2) for the high-mass tau neutrino with the extra energy is $0.47 \mathrm{EeV}$ in the good agreement with the observed 0.6 and $0.56 \mathrm{EeV}[2]$ [3].

\section{The Periodic Table of Elementary Particles for Baryonic Matter and Dark Matter and the Cosmic Rays}

In the ESM to include baryonic matter, dark matter, and gravity, the periodic table of elementary particles for baryonic matter, dark matter, and gravity is based on the seven principal mass dimensional orbitals for stable baryonic matter leptons (electron and left-handed neutrinos), gauge bosons, gravity, and dark matter and the seven auxiliary mass dimensional orbitals for unstable leptons (muon and tau) and quarks [10] [11] as in Figure 3 and in Table 3.

The masses of unstable leptons and quarks ( $\mathrm{d}=7$ and 8$)$ on the auxiliary mass dimensional orbitals are derived from the masses of electron $\left(\mathrm{F}_{6}\right), \mathrm{B}_{6}$, and $\mathrm{B}_{7}$ [12], so the masses on the auxiliary mass dimensional orbitals are derived from the masses on the principal mass dimensional orbitals. Fractional charge quarks are on the auxiliary mass dimensional orbitals, and have $\mathrm{SU}(3)_{\mathrm{c}}$ to force the fractional charge quarks to form integral charge composites of quarks [12] [18]. No isolated free fractional charge quark is allowed. The force is the strong force. The periodic table of elementary particles contains three generations of leptons $\left(\mathrm{e} / v_{\mathrm{e}}\right.$, $\mu / v_{\mu}$ and $\left.\tau / v_{\tau}\right)$ and quarks $(\mathrm{u} / \mathrm{d}, \mathrm{s} / \mathrm{c}$, and $\mathrm{b} / \mathrm{t})$, five dark matter leptons $\left(v_{\mathrm{DM} 5}, v_{\mathrm{DM} 6}\right.$,

Stable Baryonic Matter Leptons

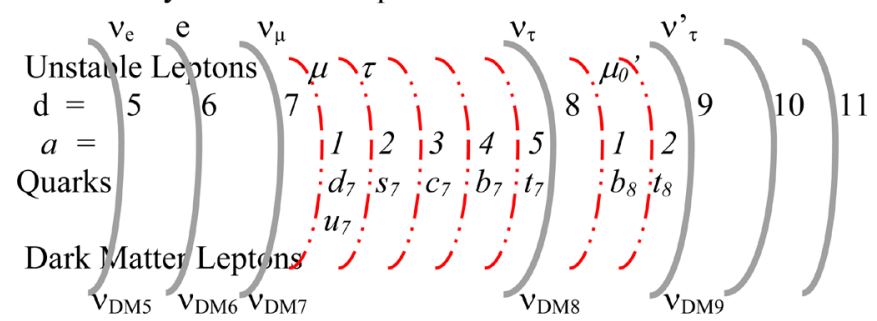

Figure 3. Leptons and quarks in the seven principal mass dimensional orbitals (solid lines) denoted by the principal mass dimensional orbital number $\mathrm{d}$ and the seven auxiliary dimensional mass orbitals (dash-dotted lines) denoted by the auxiliary mass dimensional orbital number $a$. 
Table 3. The periodic table of elementary particles for baryonic matter and dark matter.

\begin{tabular}{|c|c|c|c|c|c|c|c|c|c|c|}
\hline \multirow{2}{*}{$\mathrm{d}$} & $\mathrm{a}=0$ & $a=0$ & 1 & 2 & 1 & 2 & 3 & 4 & 5 & $a=0$ \\
\hline & $\underline{\text { Stable Baryonic Matter Leptons }}$ & $\underline{\text { Dark Matter Leptons }}$ & Unstable Leptons & & & larks & & & & $\underline{B o s o n s}$ \\
\hline 5 & $v_{\mathrm{e}}$ & $v_{\mathrm{DM} 5}$ & & & & & & & & $\mathrm{~B}_{5}=\mathrm{A}$ electromagnetism \\
\hline 6 & $\mathrm{e}$ & $v_{\mathrm{DM} 6}$ & & & & & & & & $\mathrm{~B}_{6}=\pi_{1 / 2}$ strong \\
\hline 7 & $v_{\mu}$ & $v_{\mathrm{DM} 7}$ & $\mu$ & $\tau$ & $\mathrm{d}_{7} / \mathrm{u}_{7}$ & $\mathrm{~s}_{7}$ & $\mathrm{c}_{7}$ & $\mathrm{~b}_{7}$ & $\mathrm{t}_{7}$ & $\mathrm{~B}_{7}=Z_{L}^{0}$ left-handed weak \\
\hline 8 & $v_{\tau}$ & $v_{\mathrm{DM} 8}$ & $\mu_{0}^{\prime} \quad$ (hidden) & & $\mathrm{b}_{8}$ (hidden) & $\mathrm{t}_{8}$ & & & & $\mathrm{~B}_{8}=\mathrm{X}_{\mathrm{R}}$ right-handed CP \\
\hline 9 & $v_{\tau}^{\prime}\left(\right.$ high-mass $\left.v_{\tau}\right)$ & $v_{\mathrm{DM} 9}$ & & & & & & & & $\mathrm{~B}_{9}=\mathrm{X}_{\mathrm{L}}$ left-handed CP \\
\hline 10 & & & & & & & & & & $\mathrm{~B}_{10}=Z_{R}^{0}$ right-handed weak \\
\hline 11 & & & & & & & & & & $\mathrm{~B}_{11}=$ gravity \\
\hline
\end{tabular}

$\mathrm{d}=$ principal mass dimensional orbital number, $\mathrm{a}=$ auxiliary mass dimensional orbital number.

$v_{\mathrm{DM} 7}, v_{\mathrm{DM} 8}$, and $\left.v_{\mathrm{DM} 9}\right)$, six gauge bosons, and gravity. The standard model Higgs boson is the composite of the extra-muon $\mu^{\prime}$ and anti-extra muon $\bar{\mu}^{\prime}$ in Table 3 [12] [19]. As the parts of the standard model Higgs boson and the BDM Higgs boson, the two baryonic leptons $\mu_{0}^{\prime}$ and $v_{\tau}^{\prime}$, respectively, are outside of the three generations of leptons and quarks.

The periodic table of elementary particles calculates accurately the particle masses of all leptons, quarks, gauge bosons, the Higgs boson, and the cosmic rays by using only five known constants: the number (seven) of the extra spatial dimensions in the eleven-dimensional membrane, the mass of electron, the masses of $\mathrm{Z}$ and $\mathrm{W}$ bosons, and the fine structure constant [12] [13]. The calculated masses are in excellent agreements with the observed masses. For examples, the calculated masses of muon, top quark, pion, neutron, and the standard model Higgs boson are 105.55 MeV, 175.4 GeV, 139.54 MeV, 939.43 MeV, and $126 \mathrm{GeV}$, respectively, in excellent agreements with the observed $105.65 \mathrm{MeV}$, 172.4 GeV, $139.57 \mathrm{MeV}, 939.27 \mathrm{MeV}$, and $126 \mathrm{GeV}$, respectively. The calculated mass of $B_{11}$ in Table 3 is $1.13 \times 10^{19} \mathrm{GeV}$ in good agreement with the Planck mass $1.22 \times 10^{19} \mathrm{GeV}$ for gravity.

The periodic table of elementary particles calculates accurately the values of the UHE baryonic matter downward-pointing cosmic rays in terms of the knees-ankles-toe as described in the previous paper [13]. The energy spectrum from $10^{9} \mathrm{eV}$ to $10^{20} \mathrm{eV}$ appears to follow a single power law except few breaks at the knees-ankles-toe [20]. The power index increases at the first knee and the second knee, and decreases at the ankle. Above $4 \times 10^{19} \mathrm{eV}$, the power index increases as the "toe" as the last power index increase below the GZK limit $(5 \times$ $10^{19} \mathrm{eV}$ ) as the upper limit of the cosmic rays [16] [17]. The fine structure of the cosmic ray spectrum [21] shows that an ankle with decrease in power index is in between the first knee and the second knee, resulting in two knees, two ankles, and one toe. In the periodic table, some high-mass dimensional fermions $\left(F_{d}\right.$ where $d=$ mass dimensional orbital number from 5 to 11$)$ and bosons $\left(B_{d}\right)$ are involved in the knees-ankles-toe. At the knees and the toe, some parts of the 
energies from the energy sources of cosmic rays are spent to generate $F_{d}$ and $B_{d}$, resulting in the increase of power index. The ankles are the the middle points (midpoints) between the adjacent dimensional fermions and bosons. At a midpoint, the energy is too high to keep the thermally unstable high-mass dimensional particle, resulting in the decay and the decrease of power index. The midpoint is expressed as follows.

$$
M_{\text {midpoint }}=\exp \left(\left(\ln \left(M_{\text {adjacent dimensional ferion }}\right)+\ln \left(M_{\text {adjacent dimensional boson }}\right)\right) / 2\right)
$$

The calculations of the the knees-ankles-toe are in Table 4. The calculated masses of $\mathrm{B}_{8}$, the midpoint, $\mathrm{F}_{9}\left(=v_{\tau}\right)$, the midpoint, and $\mathrm{B}_{9}$, are $1.7 \times 10^{15}, 2 \times$ $10^{16}, 2.4 \times 10^{17}, 2.8 \times 10^{18}$, and $3.2 \times 10^{19} \mathrm{eV}$, respectively, which are in good agreement with observed $3 \times 10^{15}, 2 \times 10^{16}, 3 \times 10^{17}, 3 \times 10^{18}$, and $4 \times 10^{19} \mathrm{eV}$ for the first knee, the first ankle, the second knee, the second ankle, and the toe, respectively as in Table 4 . The observed second knee is for the formation of $\mathrm{F}_{9}$ which is the high-mass tau neutrino $v_{\tau}^{\prime}$ corresponding to the observed high-mass tau neutrino without the extra energy in the upward-going ANITA events.

\section{Summary}

This paper posits that the upward-going ANITA events are derived from the cosmic ray of the baryonic-dark matter (BDM) Higgs boson that survives the passage through the Earth. In the extended standard model (ESM) for baryonic matter and dark matter, the spontaneous symmetry breaking through the Higgs mechanism for the symmetrical massless baryonic matter left-handed neutrinos and massless dark matter right-handed neutrinos produced massless baryonic matter left-handed neutrinos, sterile massive dark matter neutrinos, and the BDM Higgs boson. (Dark matter particles are the massive neutrinos.) This paper proposes the BDM Higgs boson is the composite high-mass tau neutrino and the high-mass dark matter neutrino. Other than gravity, sterile dark matter does not undergo any interaction with baryonic matter. Meanwhile, dark matter is incompatible to dense baryonic matter, because the incompatibility explains the failure to detect dark matter by the contact (interaction) between dark matter and

Table 4. The calculated masses for dimensional bosons-fermions and the observed $\mathrm{eV}$ for the knees-ankles-toe.

\begin{tabular}{|c|c|c|c|c|}
\hline $\mathrm{B}_{\mathrm{d}}, \mathrm{F}_{\mathrm{d}}$ & calculated eV & Calculation & cosmic rays & observed eV \\
\hline $\mathrm{B}_{8}$ & $1.7 \times 10^{15}$ & $\mathrm{M}_{\mathrm{Z}} / \alpha^{2}$ & the first knee & $3 \times 10^{15}$ \\
\hline The midpoint between $\mathrm{B}_{8}$ and $\mathrm{F}_{9}$ & $2 \times 10^{16}$ & Equation (6) & the first ankle & $2 \times 10^{16}$ \\
\hline $\mathrm{F}_{9}=v_{\tau}^{\prime}$ (high-mass tau neutrino) & $2.35 \times 10^{17}$ & $\mathrm{M}_{\mathrm{Z}} / \alpha^{3}$ & the second knee & $3 \times 10^{17}$ \\
\hline The midpoint between $\mathrm{F}_{9}$ and $\mathrm{B}_{9}$ & $2.8 \times 10^{18}$ & Equation (6) & the second ankle & $3 \times 10^{18}$ \\
\hline $\mathrm{B}_{9}$ & $3.2 \times 10^{19}$ & $\mathrm{M}_{\mathrm{Z}} / \alpha^{4}$ & the toe & $4 \times 10^{19}$ \\
\hline $\mathrm{F}_{10}$ & $4.4 \times 10^{21}$ & $\mathrm{M}_{\mathrm{Z}} / \alpha^{5}$ & beyond the GZK limit $\left(5 \times 10^{19} \mathrm{eV}\right)$ & not observed \\
\hline $\mathrm{B}_{11}$ & $1.13 \times 10^{28}$ & $\mathrm{M}_{\mathrm{Z}} / \alpha^{8}$ & Plank mass for gravity & $1.22 \times 10^{28}$ \\
\hline
\end{tabular}

$\mathrm{B}_{\mathrm{d}}=$ mass dimensional orbital boson, $\mathrm{F}_{\mathrm{d}}=$ mass dimensional orbital fermion, $\mathrm{M}_{\mathrm{Z}}=$ mass of $\mathrm{Z}$ boson, $\alpha=\alpha_{\mathrm{e}}$. 
baryonic matter on the Earth. The BDM Higgs boson is both baryonic matter and dark matter. The inactive dark matter in the BDM Higgs boson allows the BDM Higgs boson to be stable (inactive) in space, air, water, and ice. However, during the passage through the high-density part of the Earth, because of the inactivity (other than gravity) and incompatibility between dark matter and dense baryonic matter, the BDM Higgs boson is transformed into the oscillating BDM Higgs boson between the composite of the high-mass tau neutrino and the high-mass dark matter neutrino and the composite of the high-mass tau neutrino and the low-mass dark matter neutrino to maintain stability (inactivity) with the maximum dark matter and to minimize incompatibility with the minimum dark matter. Near the end of the passage through the Earth, the low-density water-ice layer of the Earth allows the dark matter in the oscillating BDM Higgs boson to escape, so the oscillating BDM Higgs boson decays into the high-mass tau neutrino with the extra energy and the incompatible low-mass dark matter neutrino $(27 \mathrm{eV})$ which is easier to escape than the high-mass dark matter neutrino. The high-mass tau neutrino is converted into ultra-high-energy tau neutrino which decays into tau lepton through the charged-current interactions, and tau lepton emerges from the surface of ice. Based on the periodic table of elementary particles, the calculated mass for the high-mass tau neutrino with the extra energy is $0.47 \mathrm{EeV}$ in the good agreement with the observed 0.6 and 0.56 $\mathrm{EeV}$. The decay products of tau lepton were detected by the ANITA.

In the periodic table of elementary particles, the seven extra spacetime dimensions in the 11-spacetime dimensional membrane in M-theory are in the form of the seven mass dimensional orbitals for all internal symmetries. The periodic table of elementary particles for baryonic matter, dark matter, and gravity is based on the seven principal mass dimensional orbitals for stable baryonic matter leptons (electron and left-handed neutrinos), gauge bosons, gravity, and dark matter and the seven auxiliary mass dimensional orbitals for unstable leptons (muon and tau) and quarks. The last principal mass dimensional orbital is for gravity.

The formation of the periodic table of elementary particles involves the three steps. The first step is the BDM spontaneous symmetry breaking through the Higgs mechanism for the symmetrical five massless baryonic matter left-handed neutrinos and five massless dark matter right-handed neutrinos on the principal mass dimensional orbitals to produce massless baryonic matter left-handed neutrinos, massive dark matter neutrinos, and the massive BDM Higgs boson. The second step is the addition of electromagnetism as $U(1)$ and the standard model spontaneous symmetry breaking $\left(\mathrm{SU}(2)_{\mathrm{L}} \times \mathrm{U}(1)_{\mathrm{Y}} \rightarrow \mathrm{U}(1)_{\mathrm{EM}}\right)$ through the Higgs mechanism to produce massless neutrinos-massive weak bosons, massive electron-massless photon, and the standard model Higgs boson. The third step is the addition of the seven auxiliary mass dimensional orbitals for unstable leptons (muon and tau) and quarks to form the periodic table of elementary particles.

The periodic table of elementary particles for baryonic matter, dark matter, and gravity calculates accurately the masses of all elementary particles and the 
cosmic rays by using only five known constants. The periodic table of elementary particles calculates accurately the values of the UHE baryonic matter downward-pointing cosmic rays in terms of the knees-ankles-toe. The calculated value of the second knee is $2.35 \times 10^{17} \mathrm{eV}$ in good agreement with $3 \times 10^{17} \mathrm{eV}$ for the observed second knee which is for the formation of the high-mass tau neutrino, corresponding to the observed high-mass tau neutrino without the extra energy in the upward-going ANITA events.

\section{Conflicts of Interest}

The author declares no conflicts of interest regarding the publication of this paper.

\section{References}

[1] Gorham, P., et al. [ANITA Collaboration] (2009) The Antarctic Impulsive Transient Antenna Ultra-High-Energy Neutrino Detector Design, Performance, and Sensitivity for 2006-2007 Balloon Flight. Astroparticle Physics, 32, 10-41. https://doi.org/10.1016/j.astropartphys.2009.05.003

[2] Gorham, P., et al. [ANITA Collaboration] (2016) Characteristics of Four Upward-Pointing Cosmic-Ray-Like Events Observed with ANITA. Physical Review Letters, 117, 071101. https://doi.org/10.1103/PhysRevLett.117.071101

[3] Gorham, P., et al. [ANITA Collaboration] (2018) Observation of an Unusual Upward-Going Cosmic-Ray-Like Event in the Third Flight of ANITA. arXiv:1803.05088 [astro-ph.HE]

[4] Cherry, J. and Shoemaker, I. (2018) A Sterile Neutrino Origin for the Upward Directed Cosmic Ray Shower Detected by ANITA. arXiv:1802.01611 [hep-ph]

[5] Huang, G. (2018) Sterile Neutrinos as a Possible Explanation for the Upward Air Shower Events at ANITA. Physical Review D, 98, 043019. https://doi.org/10.1103/PhysRevD.98.043019

[6] Boyle, L., Finn, K. and Turok, N. (2018) The Big Bang, CPT, and Neutrino Dark Matter. arXiv:1803.08930 [hep-ph]

[7] Anchordoqui, L., et al. (2018) Upgoing ANITA Events as Evidence of the CPT Symmetric Universe. Letters in High Energy Physics, 1, 13. https://doi.org/10.31526/LHEP.1.2018.03

[8] Fox, D. (2018) The ANITA Anomalous Events as Signatures of a Beyond Standard Model Particle, and Supporting Observations from IceCube. arXiv:1809.09615 [astro-ph.HE]

[9] Chung, D. (2014) Galaxy Evolution by the Incompatibility between Dark Matter and Baryonic Matter. International Journal of Astronomy and Astrophysics, 4, 374-383. https://doi.org/10.4236/ijaa.2014.42032

[10] Chung, D. (1998) The Masses of Hadrons. Speculations in Science and Technology, 21, 277-289. https://doi.org/10.1023/A:1005513404873

[11] Chung, D. (2014) The Periodic Table of Elementary Particles Based on String Theory. Journal of Modern Physics, 5, 1234-1243. https://doi.org/10.4236/jmp.2014.514123

[12] Chung, D. (2016) The Accurate Mass Formulas of Leptons, Quarks, Gauge Bosons, the Higgs Boson, and Cosmic Rays. Journal of Modern Physics, 7, 1591-1606. https://doi.org/10.4236/jmp.2016.712144

[13] Chung, D. (2014) The Knees-Ankles-Toe in Cosmic Rays and the Periodic Table of 
Elementary Particles, Journal of Modern Physics, 5, 1467-1472. https://doi.org/10.4236/jmp.2014.515148

[14] Chung, D. and Krasnoholovets, V. (2013) The Light-Dark Dual Universe for the Big Bang and Dark Energy. Journal of Modern Physics, 4, 77-84.

https://doi.org/10.4236/jmp.2013.47A1009

[15] Jarosik, N., et al. (2011) Seven-Year Wilkinson Microwave Anisotropy Probe (Wmap ${ }^{*}$ ) Observations: Sky Maps, Systematic Errors, and Basic Results. The Astrophysical Journal Supplement Series, 192, 14. https://doi.org/10.1088/0067-0049/192/2/14

[16] Greisen, K. (1966) End to the Cosmic Ray Spectrum? Physical Review Letters, 16, 748-750. https://doi.org/10.1103/PhysRevLett.16.748

[17] Zatsepin, G. and Kuzmin, V. (1966) Upper Limit of the Spectrum of Cosmic Rays. Letters to Journal of Experimental and Theoretical Physics, 4, 78-80.

[18] Chung, D. (2016) The Integer-Fraction Principle of the Digital Electric Charge for Quarks and Quasiparticles. Journal of Modern Physics, 7, 1150-1159.

https://doi.org/10.4236/jmp.2016.710104

[19] Chung, D. and Hefferlin, R. (2013) The Higgs Boson in the Periodic System of Elementary Particles. Journal of Modern Physics, 4, 21-26.

https://doi.org/10.4236/jmp.2013.44A004

[20] Drury, L. (2012) Origin of Cosmic Rays. Astroparticle Physics, 39-40, 52-60. https://doi.org/10.1016/j.astropartphys.2012.02.006

[21] Sveshnikova, L., et al. (2013) Nearby Sources in the Transition Region between Galactic and Extragalactic Cosmic Rays. Journal of Physics: Conference Series (JPCS), 409, 012062. https://doi.org/10.1088/1742-6596/409/1/012062 